\title{
Optical Imaging of the Central Kiloparsecs of a Sample of Low Luminosity AGN
}

\author{
M.J. Marchã and S. Antón \\ OAL Portugal \& Jodrell Bank, U.K.
}

\begin{abstract}
Selected with the primary objective of studying the differences between BL Lacs and their closest relatives, a sample of 55 optically bright, low luminosity core dominated radio sources has been studied. The spectroscopy carried out for this sample, however, yielded not only BL Lacs but also a completely unexpected number of sources with strong emission lines (much like those found in Sy galaxies), and almost $50 \%$ of galaxies with weak ( $\mathrm{EW} \leq 30 \AA$ ), or absent emission lines. Such optical diversity is at odds with the traditional unification schemes for low luminosity AGN and it has prompted an optical imaging campaign. High resolution optical images obtained at NOT are discussed, in particular the central colours and profiles of the different types of sources found in the sample.
\end{abstract}

The optical diversity found among the objects of a sample of radio-loud sources selected with similar radio properties is at odds with the current unified schemes for the low-luminosity radio-loud regime. This diversity ranges from Passive Elliptical Galaxies (PEGs) with their weak emission lines and typical stellar absorption features, through BL Lacs and BL Lac candidates with their weak or absent emission lines, to Sy-like sources with strong emission lines in their spectra. Such optical variety among sources with similar radio properties prompts the question of whether there is a correlation between the optical activity and the large scale phenomena.

Using imaging data obtained at the Nordic Optical Telescope (NOT), the host galaxies and the central colours of the different sources were investigated. The results can be summarised as follows:

\section{- Host Galaxies}

The host galaxies of the different types of objects are well fitted by a de Vaucouleurs profile plus a scaled PSF. The contribution of the PSF component decreases from BL Lacs and Sy-like to BL Lac candidates, and becomes weakest in PEGs.

\section{- Central Colours}

All BL Lacs and Sy-like objects have bluer central regions, whereas none of the $3 \mathrm{BL}$ Lac candidates with data showed a bluer central region. All PEGs showed a red central region which is a common feature of normal galaxies. 

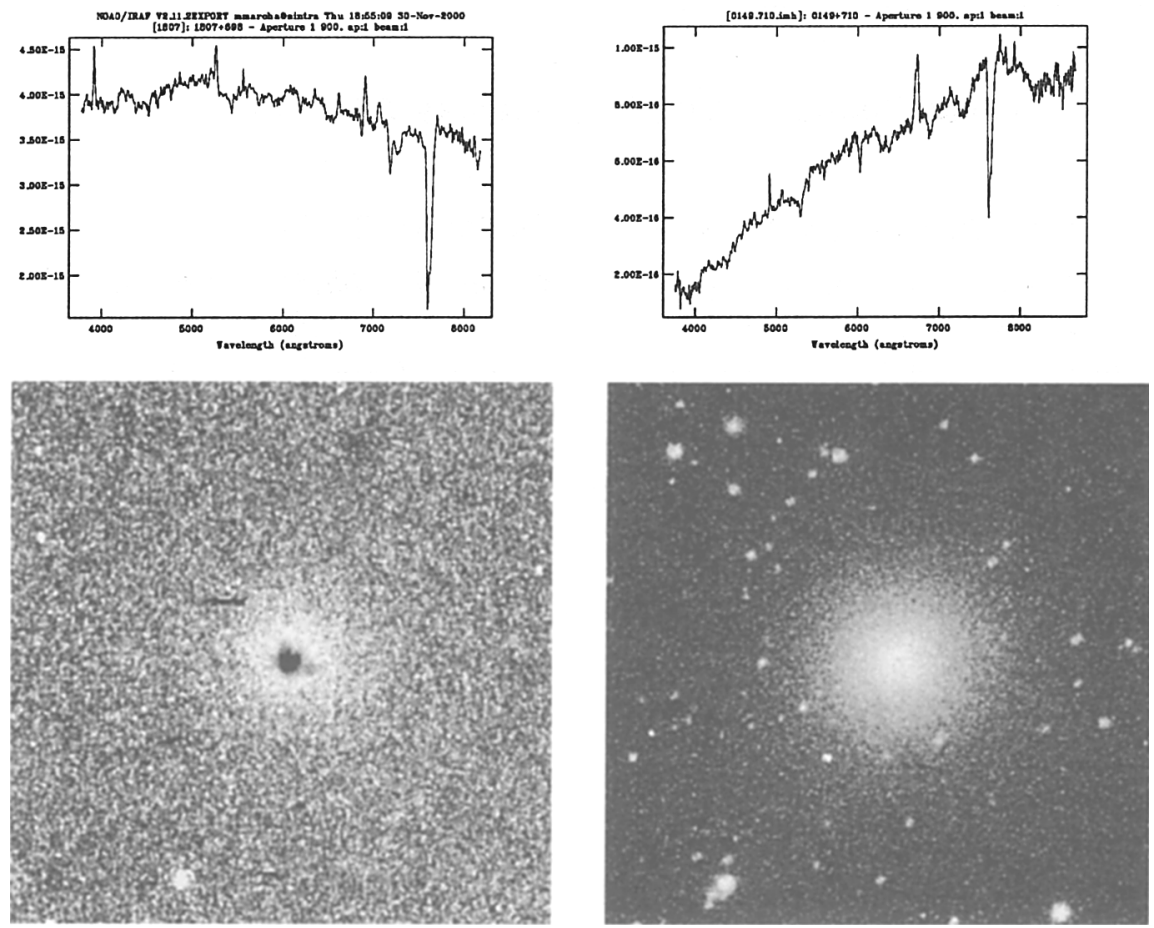

Figure 1. Left - BL Lac 1807+698. Right - BL Lac candidate $0149+710$.

Based on these observations it is possible to conclude that:

1. The angle independent properties are very similar in BL Lacs, BL Lac candidates, Sy-Like and PEGs.

2. BL Lacs and Sy-like show bluer central regions indicating that there is an extra component: non-thermal in BL Lacs and possibly a sum of nonthermal plus thermal accretion disk emission in the Sy-like sources.

3. BL Lac candidates and PEGs do not show strong signs of an extra component, thus suggesting that the non-thermal emission has a low frequency cutoff.

4. The properties of the central engine of low-luminosity, radio-loud AGN seem to be independent of the host galaxy. 